\title{
VISIBLE LIGHT COMMUNICATION FOR AUDIO SYSTEMS
}

\author{
Grantham Pang, Ka-Lim Ho, Thomas Kwan, Edward Yang
}

\begin{abstract}
ABSTRAC' I
This paper describes an audio system that is wellsuited for use in a small confined area with many audio transmitters broadcasting different audio signals. The transmitter of the proposed system is constructed using visible light LEDs, in which current fed to the LEDs is modulated and encoded with audio information or messages. The audio system provides audio signal transmission in a free space optical link. The receiver, combined with an ear jack, is located at some distance from the transmitters.
\end{abstract}

The handheld receiver is designed to demodulate the optically transmitted audio information and reproduce the messages with the ear jack. lior modulating emission of ILDs, an oscillator is used to vary the frequency of on/off periods of the LEDs. The frequency of flicker is high enough to be indistinguishable by human eye and hence the L.EDs appear to be constantly illuminated.

Keywords: audio systems, light emitting diodes; audio broadcasting.

\section{Introduction}

This paper relates to an audio system that relies on visible light for transmitting audio information to a receiver located some distance away from the system. The basic idea is based on the fact that light cmitting diodes (LEDs) are semiconductor devices and are capable of fast switching with the addition of appropriate electronics. That is, the visible light emitted by the LEDs can be modulated and encoded with audio information. LEDs can then be used as a communication device for the transmission of audio information.

Optical signals have been used for communication and information processing in the field of fiber optics for many years. In this paper, a different use of optical signals for communication is described.

Contact person: Dr. Grantham Pang I)ept. of Hec. \& I Acctronic linginecring, The University of Hong Kong,Pokfulam Road, Hong Kong. 'Tel: (852)-2857-8492 lax: (852)-2559-8738 I imail: gpang@hkucechku.hk URL:htı://www.ece.hku.hk/ gpang
The proposed system depends on a direct line of sight between the transmitter and the receiver. This characteristic can be turned into an advantage in situations where a household has many appliances with audio output such as TV, PC, Hi-fi, radio and phone (Figure 1). Some or all of them could be turned on at the same time and there could be more than one people working in the same confined area. This type of home environment with many audio sources is very common in many congested cities in the world. Infrared transmission cannot solve the problem completely duc to the interference and the switching of channels is very inconvenient. The proposed system allows the user to hear from one audio source when the receiver is pointing at that appliance. Multiple users can also work in the same compact environment and the problem due to noise nuisance can be greatly reduced.

There has been rapid advance in the LED technology in recent years. Today, visible light LEDs can reach a luminous efficacy beyond 15 $1 \mathrm{~m} / \mathrm{W}$, which is typical for incandescent lamps $[1,2]$. The main idea of this paper is to modulate the visible light emitted from the LEDs for communication purpose in open space [3]. I lence, an audio system can make use of ordinary LEDs or LED dot matrix display (as used in an radio alarm clock) to provide audio broadcasting. It makes use of the visible portion of the LM spectrum, which is unregulated by $\mathrm{FCC}$ or any countries in the world.

The work reported in this paper differs from the use of infrared (IR) radiation as a medium for shortrange wireless communications $[4,5]$. Currently, infrared links and local-area networks are available. IR transceivers for use as IR data links are commercially available at cconomic prices. Comparison between the infrared and other media such as radio and microwave is given in [6]. Somehow, there has been very little work on the use of visible light as a communication medium for open-space, short-range transmission. Recent availability of high brightness LEDs makes the visible light medium even more feasible now. Lssentially, all appliances with visible LED components can utilize the visible light as an information beacon. This paper focuses on its use

Contributed Paper 
for audio transmission.

\section{Description of the audio information system}

The audio system includes a transmitter and a companion receiver. The transmitter makes use of L.EI)s or L.EI) display for audio broadcasting. It consists of an audio signal source that provides the modulated audio signal for transmission by the display assembly. The key component of the transmitter is a modulator, which includes a voltage-controlled oscillator $(\mathrm{VCO})$ for varying the switching frequency of light cmitting diodes on the dot matrix display. The on/off frequency is high enough such that the perceivable light appears to be constantly illuminated to the human eye. Hence, the transmitter provides a modulated audio signal by frequency modulation (FM).

The receiver makes use of a photo-detector to detect high frequency variations in the light flux. This detected signal is passed to appropriate amplifying and detecting stages to reproduce audio signal transmilted. Both the transmitter and the receiver require low power supply, which increases transportability of the system. As the majority of the eircuitry is T'T1, based, power supply requirements are in the vicinity of five volts.

Referring to Figure 2, the audio signal from the TV, $\mathrm{Hi}-\mathrm{fi}, \mathrm{P} \mathrm{C}$ or phone is input to the transmitter. The audio signal may have small amplitude and amplification is necessary. The audio amplificr is used to amplify the weak audio signal and to shift the average voltage level of the audio signal to an appropriate level so that the signal is suitable for input to the voltage controlled oscillator ( $\mathrm{VCO}$ ).

Incoming audio signal modulates the VCO and gencrates $\mathrm{lM}$ signal at the output, according to change in voltage level of the signal. $A$ VCO chip is used. A square wave VCO is used instead of sine wave because there are only two states (ON and $\mathrm{OFF}$ ) for the LEDs. The carrier frequency is set at $100 \mathrm{KHz}$ with a maximum frequency deviation of $\pm 50 \mathrm{KII} \%$. The modulated signal is transmitted by the switching of the LEDs, througl a driving circuit.

The optical link cmploys intensity modulation of LEDs with direct detection. With appropriate electronics, the information in the light ray is demodulated to detect the audio signal, which is reproduced with an ear jack for the broadcasting and announcement of the audio messages or music.

A block diagram representation of the receiver design is shown in Figure 3. The eircuit of the receiver is divided into eight portions. The photodetector is used to detect modulated light signal from the transmitter and convert the signal into an clectrical signal. The limiting pre-amplifier is used to amplify the electrical signal from the photodetector for the next stage. Pulse shaping and detecting circuits are used to reconstruct the square wave. Square wave pulses from previous output stages are differentiated and its negative trailing edges are clipped when transmitted through a diode. Next, a pulse generator is used to convert the pulses from the differentiator into sharp pulses, to be used by the integrator and envelope detector in the next stage for the demodulation of the signal. The band-pass filter is then used to smooth out the distortions from the integrator and envelope detector to produce an appropriate waveform. Finally, an audio power amplifier is used to amplify the weak signal from the band-pass filter so that the audio signal would be comfortable for hearing. $\Lambda$ speaker, head phone or ear jack can be used for the delivery of the audio messages or music sent from the transmitter. The receiver can be battery powered, or powered by any electrical system.

For appliances that have an LED dot matrix display, like a radio alarm clock with a display of the digital time, the IJVD on the display can be used for simultaneous display and audio broadcasting. It relates to a dot matrix IIID display panel or any I.ED display assembly for providing a visual signal in the form of light rays and for using the light rays to transmit information. The LED display is controlled through a hardware interface. More details on the use of dot matrix display for audio broadcasting is given in the next section.

\section{Components of a tricolor LED audio system}

In this section, a detailed description of a tricolor I.ED display for both signaling and broadcasting of audio information is described. The tricolor light cmitting diode dot matrix display system is shown in Figure 4. It relates to an LISD display panel or 
any L.ED display assembly for providing a visual signal in the form of light rays and for using the light rays to transmit information. The LLD display is linked to a computer through an interface circuit. An executive program runs on the computer for the visual display control of characters, decorative pattern or messages on the display pancl.

Figure 5 gives the block diagram of the interface circuit. The circuit is connected to the computer through the parallel port. There are seven parts for this circuit.

\section{(a) Row Counter Decode System}

This part is used to switch among the rows of the LED display. It contains a counter driven by the information signal, and a decoder for row selection.

(b) Segment Counter Decode System

This part is used to switch among the segments of the LED display. For segment, it means a particular color of a particular character. $\Lambda$ gain, it contains a counter, driven by a fast oscillator, and a decoder for segment selection.

(c) Pattern Storage System

This part is used to store the IED display pattern of evcry row and every segment of each character. SRAM is used in this system.

(d) Address Comparison Unit

This part compares the system-generated address ( based on the two counter systems ) against the address sent out by the computer. This is used to ensure that the SR $\triangle M$ data and display will not be disrupted until the I.EDs are in the inactive state (off state). The data is actually written to the SRAM so that it can be displayed in the next scan. This part contains a bit comparator and a register. This register is to hold the data until the system is ready to write this piece of data to the SRAM.

(e) Status Indicator

This part contains a D-type flip-flop with Asynchronous inputs of Preset and Clear. The noninverted flip-flop output pin ( strobe pin ) is used for indication of a successful write. On such occasion, this pin will be set high and enables the computer so send next data.

(f) LED Display Unit

This is the part to store temporarily the IED information and hold the LEDs in place. It should be noted that the common (row) signal is negative and segment signals are positive. This unit also contains registers to isolate the LEDs with the circuit. They can also hold temporarily information that will be sent to drive the LEDs. These are used to defer updating of data when LEDs are active and ensure that it is done when LEDs are off.

(g) Parallel Port

This is the outlet of the computer system and acts as the interface to the hardware electronics. Data and control signals are sent out from this port and strobe (status) information is delivered to the computer via this port also.

Figure 6 gives a schematic of a tricolor LED dot matrix array. In order for a light emitting diode to be on, the corresponding row signal should be high and associated segment selection signal should be low.

\section{Features of the LED display}

\subsection{Pattern update process}

'The data is first latched into the register. However, it is not sent to the SRAM data input pins and the segment registers' input pins so as not to disrupt the normal LED display process. The address is transmitted to one of the input pins of the comparator. After the comparator is enabled, this address is compared with the system-generated address. As they are matehed, the output of the comparator will be driven low. This will put the data stored in the register to the input data pins of the SRAM. The matched address is already loaded in the address pins of the SR $\Lambda \mathrm{M}$. The matched event will trigger the write enable pin of the SRAM, causing the data to be written to the SRAM at that particular address. Another pin affected is the strobe pin at the output of a flip-flop. The pin is enabled high by the event, indicating the computer that the write has been successful and it could continue writing the next data.

\subsection{Pattern Display Process}

The address is composed of two parts. The first part is from the information signal that specifies the row at which the I.EDs are active. The other part is from the $12 \mathrm{MHz}$ oscillator that specifies the segment for which data is to be latched to the display register. These two portions constitute the address of the data and this is loaded in the SRAM for data request. After the data is retrieved, it is loaded in the appropriate display register and it is 
displayed finally.

\subsection{Software for display and pattern update}

The software program used for the display control and pattern update of this tricolor LED dot matrix display is described next. For the write cycle, data is first put onto the bus and latched. The corresponding address is than loaded in the same bus and it is compared with the system-generated address. If they are matched, data is written in the SRAM at the corresponding address. A signal is sent back to the computer, indicating a successful write.

The special dot matrix display developed is very general. I)isplay effects such as scrolling and running is also included. $\Lambda$ special character data set used for the tricolor display has been created. Here, $6 \times 6$ characters are used on an $8 \times 8$ display so that they can be clearly distinguished when two characters are put side by side. The residue LLDs form a border around the character, which are displayed as background color.

\section{Application}

Figure 1 shows an example of the audio system where a handheld or car piece receiver is used to receive atdio information or music from a selected audio source. The indoor environment has many consumer electronics appliances and each can provide audio output. With the receiver adjusted to point to the transmitter of each appliance when the user is focusing his attention to it, appropriate audio signal is received. The switch from one audio device to another device is quick and natural. The viewing angle of the transmitter and receiver can be adjusted to provide an optimum setting for the user.

It should be mentioned that such a receiver can also be used in many other situations. Suppose an ILD or dot matrix display transmitter is installed in an exhibition hall. The information on individual display can be broadcast via the L.ED transmitter. When a visitor is looking at the I.ED display panel, he is automatically in the line of sight and thus receives audio messages about the specific announcement. Thus, the sound level of the exhibition hall can be minimized. The same receiver can also be used to reccive audio messages or music from all commercial billboards made up of I.FDs.

\section{Implementation and Results}

Iwo prolotypes of the audio information system described in this paper have been implemented in the Industrial Nutomation Laboratory at The University of 1 long Kong. ligure 7 is a photograph of the receiver. Figures 8 shows a simple LID transmitter consisting of l.EDs with a luminous intensity of $1100 \mathrm{med}$ at rated $20 \mathrm{~mA}$ driving current. It is a $5 \mathrm{~mm}$ I,I:D lamp with a 15 degree viewing angle. With just one IIID, it can be demonstrated that the transmission distance can reach $390 \mathrm{~cm}$ with a $50 \mathrm{~mm}$ focusing lens in front of the receiver photodiode. Without the lens, the transmission distance can reach $77 \mathrm{~cm}$.

Figure 9 is a photograph of a tricolor dot matrix display. The display consists of an 8 by 8 ILED dot matrix. The receiver was placed at two meters away from the display and satisfactory audio output was obtained. It should be noted that the type of tricolor LED used for the construction of the implemented dot matrix display is commonly available. I arge audio transmission range can be achieved by employing high brightness tricolor LLEDs [1,7].

The specifications of the tricolor dot matrix display is given below:

\begin{tabular}{|c|c|}
\hline Color & red, green, orange \\
\hline Construction & 64 tricolor LHISs \\
\hline Sizc & $4 \mathrm{~cm}$ square: \\
\hline Nominal usage & $5 \mathrm{~V} ; 0.24 \mathrm{~A}$ \\
\hline $\begin{array}{l}\text { Nominal power } \\
\text { consumption }\end{array}$ & $1.2 \mathrm{~W}$ \\
\hline I uminous intensity & $1 \overline{80} \overline{\mathrm{cd}}$ \\
\hline $\begin{array}{l}\text { Viewing angle } \\
20_{1 / 2} \text { (half power) }\end{array}$ & 80 degrees \\
\hline $\begin{array}{l}\text { Distance for audio } \\
\text { transmission } \\
\text { (indoors) }\end{array}$ & $\begin{array}{l}40 \overline{c m} \text { (no lens); } \\
200 \mathrm{~cm} \text { (with focusing } \\
\text { lens) }\end{array}$ \\
\hline
\end{tabular}

The radiation pattern of the panel is given in Figure 10. The viewing angle of the display is around 80 degrees. An IlP Audio Analyzer has been used to provide audio measurement for the visible light IIID audio broadcasting panel. It has a low distortion signal source with a signal analyzer. In a measurement on the signal-to-noise ratio, the result is shown in Figure 11. Figure 12 shows the 
distortion measurement on signals from 237 to 10Krlz. The frequency response characteristic of the system is shown in Figure 13.

\section{Conclusions}

An audio system made up of light emitting diode (I.ED) and L.ED dot matrix display is described. It uses the visual light rays for transmitting audio messages to a remotely located receiver. It has long been realized that visible light has the potential to be modulated and used as a communication channel with high entropy. This application makes use of free space as communication medium and the receiver is required to be in line-of-sight (LOS) with the transmitter.

Such an audio system would be very useful in situations where there are multiple audio sources in a confined area. Ilere, the direct LOS property of visible light communication is utilized. It should be noted that the transmitter provides easy target for the line-of-sight reception by the receiver. This is because the LEDs, being on all times, are also indicators of the locations of the transmitter. Two prototypes of the andio systems have been implemented and they give satisfactory quality for consumer-use audio appliances. The experimental results in this paper have demonstrated that the proposed idea is totally feasible.

\section{References}

1. G.B. Stringfellow and M.G. Craford, "High Brightness Light Timitting Diodes", Semiconductors and Semimetals Vol. 48, Academic Press, 1997.

2. M.G. Craford, "LEDs Challenge the Incandescents", IEEE Circuits and Devices, pp. 24-29, Scptember 1992.

3. P.P. Smyth, P.L. Eardley, K.T. Dalton, D.R. Wislcy, P. McKec and D. Wood, "Optical Wircless - a prognosis", Proc. On Wireless Data Transmission, SPIF: Vol. 2601, pp. 212 225, October 23-25, 1995.

4. T.S. Chu and M.J. Gans, "Fligh speed infrared local wircless communication", ILEE Communications Magazine, pp. 4-10, August 1997.

5. M. Meyer, "In(frared I LI)s", Compound Semiconductor, pp. 39-40, May/June, 1996.

6. J.M. Kahn and J.R. Barry, "Wireless Infrared Communications", Proceedings of the llikE, Vol. 85, No.2, pp.265-298, Feb 1997.

7. K. Werner, "Higher visibility for LEDs", IEEE: Spectrum, pp.30-39, July, 1994.

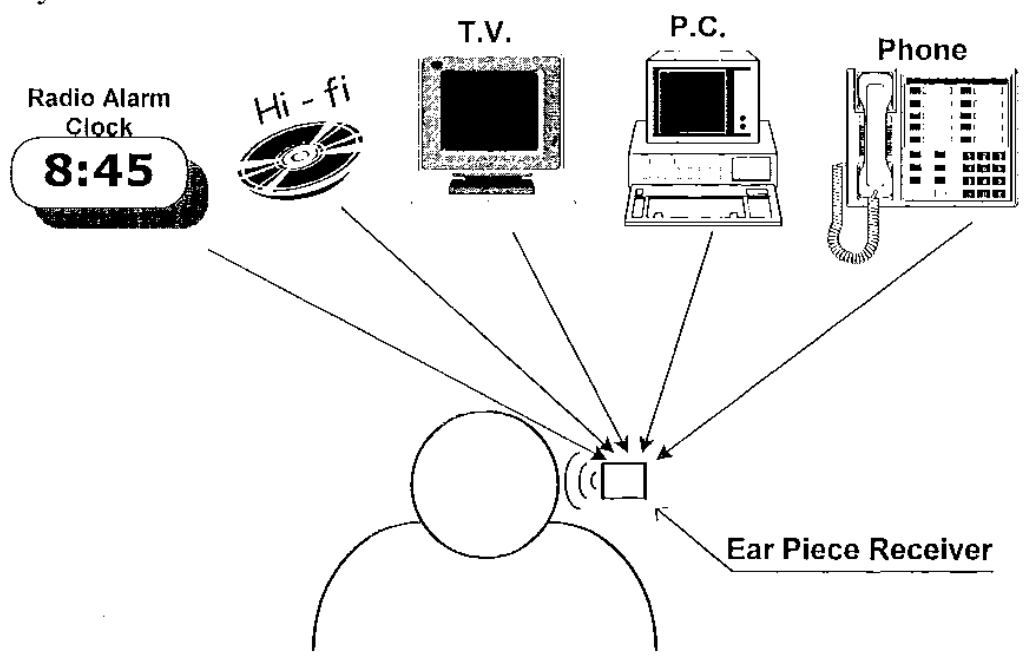

Figure 1: An audio system with multiple transmitters.

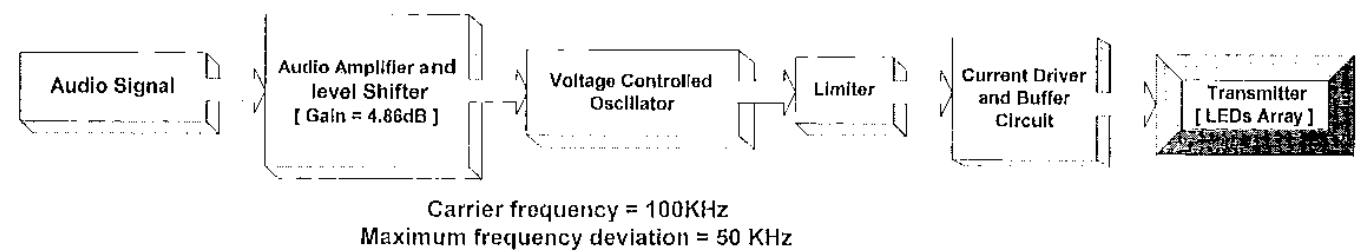

Figure 2 : Block diagram representation of the schematic diagram of the audio signal transmitter. 


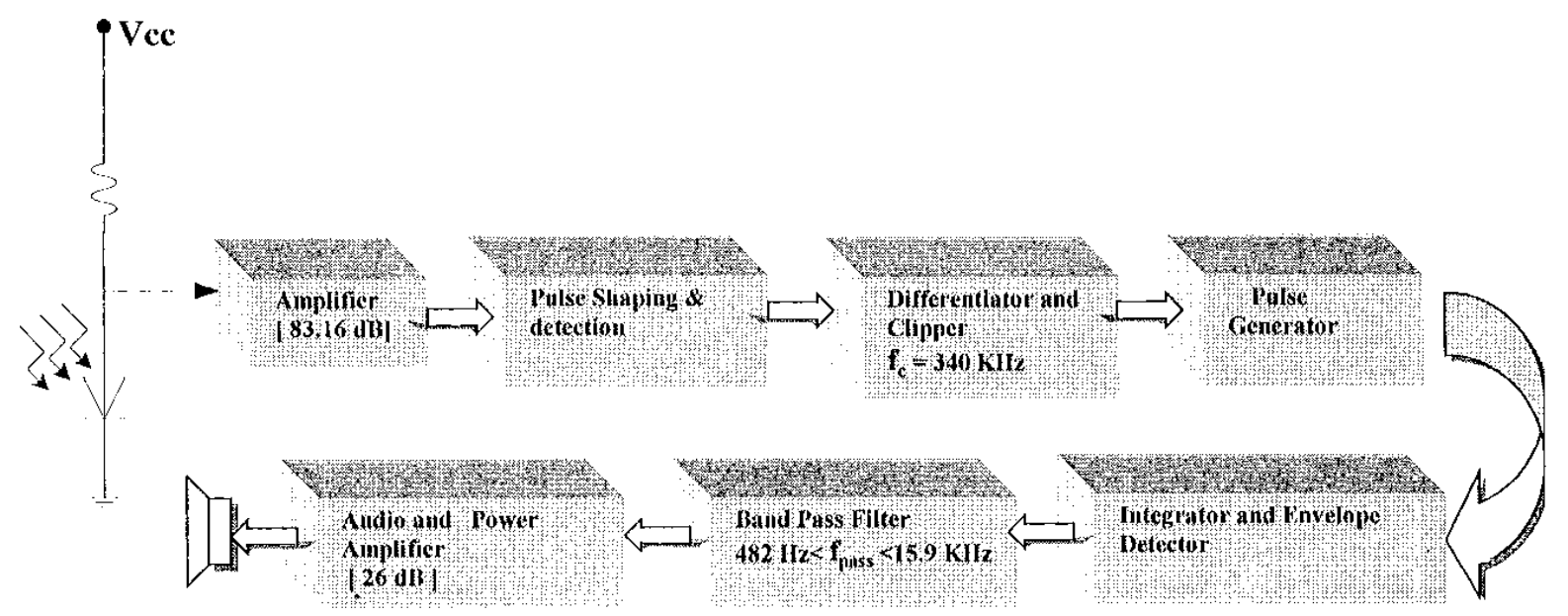

Figure 3 : Block diagram representation of the receiver.

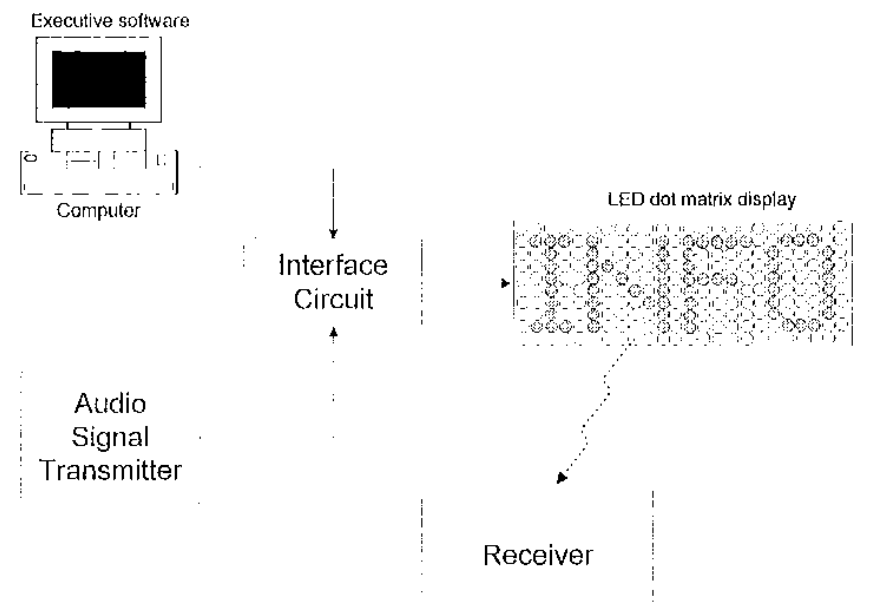

Figure 4 : Block diagram of the tricolor L,LI) dot matrix display system, an interface circuit which links the display to a computer, an audio signal transmitter, and the companion receiver.

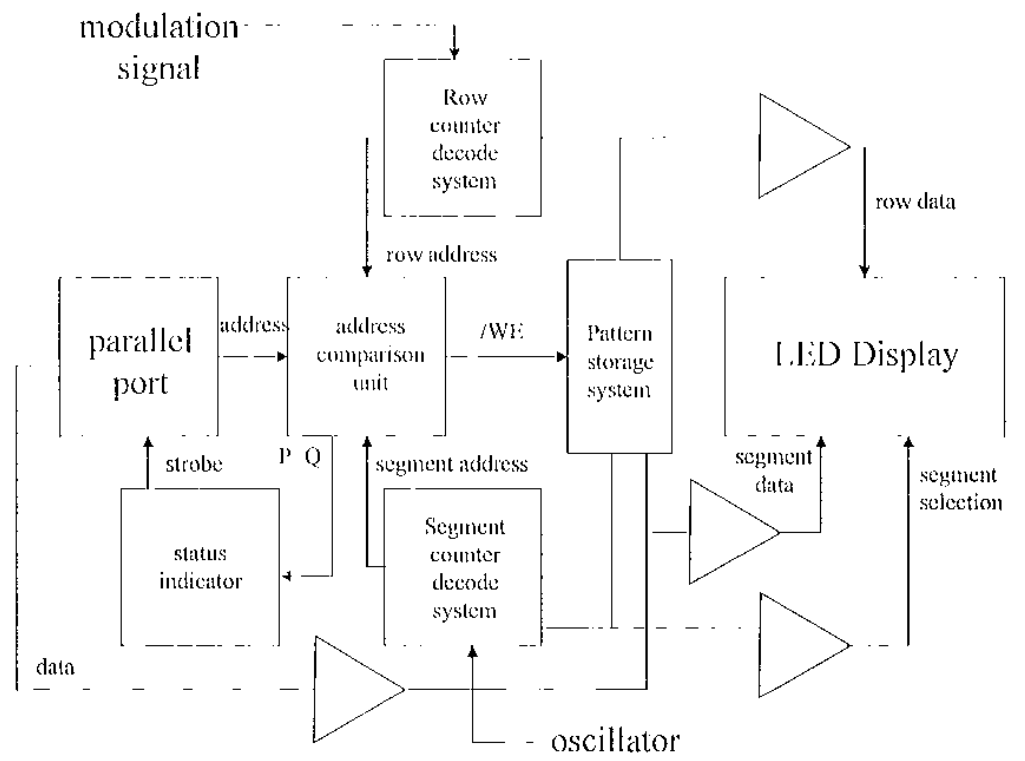

Figure 5: Circuit diagram of the interface design. 


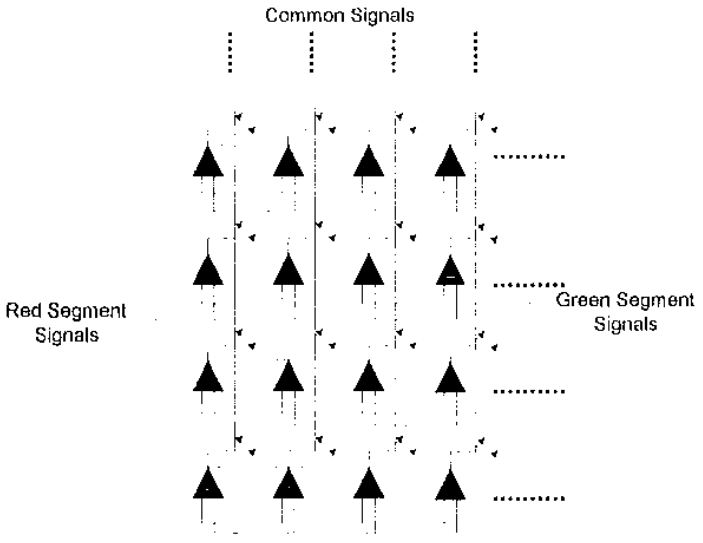

Figure 6: Schematic of a tricolor dot matrix display using light emitting diodes.

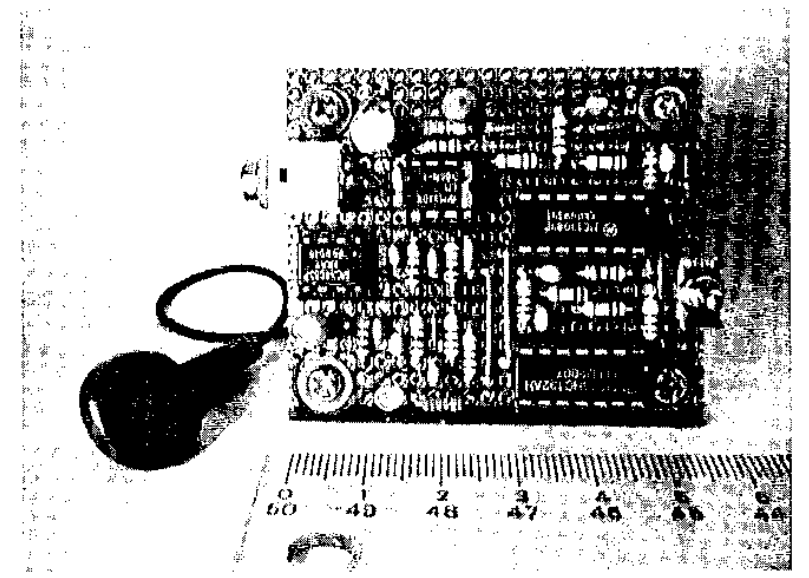

Figure 7: Photograph of a receiver.

\section{Professional Biography}

\section{Dr. Grantham K. H. Pang}

B.SC., Ph.D. (Cambridge), P. ling., C. Eng., MIEL, MIELE

Dr. Grantham K. H. Pang obtained his Ph.D. degree from the University of Cambridge in 1986 for research in multivariable control system design and expert systems. He was with the Department of Electrical and Computer

Enginecring, University of Waterloo, Canada, from 1986 to 1996 and joined the Department of Helectrical and Electronic Enginecring at The University of Hong Kong in 1996. Since 1988, he published more than 60 lechnical papers and has authored or co-authored three books. His research interests include expert systems for control system design, intelligent control, intelligent transportation system, neural networks, control theory and computer-aided design. In 1989, he was awarded the ICI Prize for author-

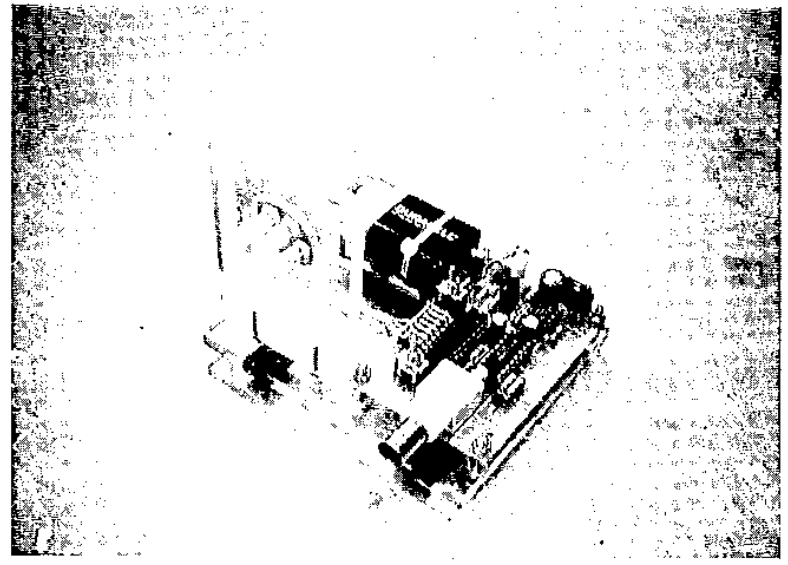

Figure 8: Photograph of the LED transmitter.

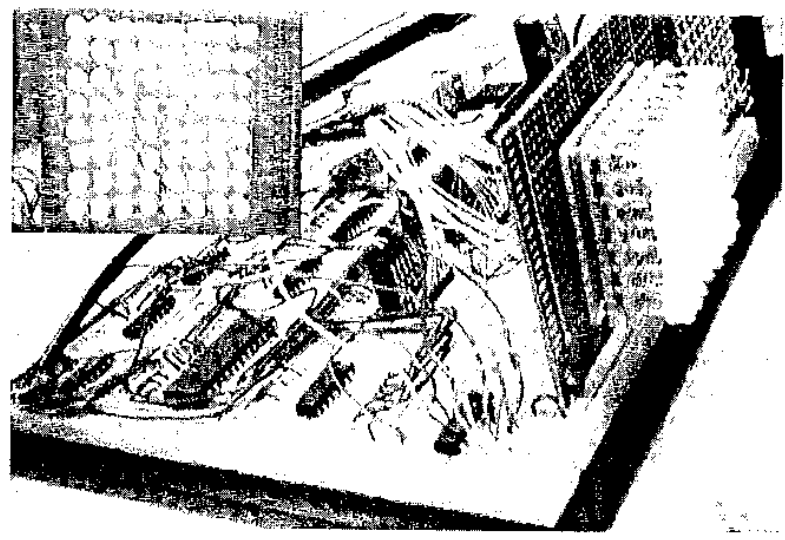

Figure 9: Photograph of the tricolor LLD dot matrix display/transmitter.

ship of the best paper on the application of the theory of control published in the Transaction of fustitute of Mcasurement and Control.Dr, Pang was the Oryanizing Chair of the 1996 IEEE Symposium on Computer-Aided Control System Design. He was appointed by the President of the Hilst: Control Systems Society as the Chair of the Technical Committec on Computer-Aided Control System Design (1993-95). In 1994, he worked as a Senior Visiting Reseatcher at Hitachi Rescarch Laboratory in Japan. Di. Pang has acted as a consultant to many companies which include Mitsubishi Electric Corp, in Japan, Northern Telecom and Imperial Oil I td. in Canada. Dr. Pang is an Editor of the International Journal of Intelligent Control and Systems. Also, he is an of the journal Control and Computers published by the International Association of Science and Technology for Development (IASTED). He is a Chartered Flectrical lingineer and a member of the IFEt, HKIL and IEHE. 